\title{
Investigation of the Operational and Technological Reliability of the Small-size Internal Threading Process
}

\author{
Aleksander Kharchenko ${ }^{1, \mathrm{a}^{*}}$, Andrey Kharchenko ${ }^{1, \mathrm{~b}}$ and Ekaterina Vladetskaya ${ }^{1, \mathrm{c}}$ \\ ${ }^{1}$ Sevastopol State University, Universitetskaya St., 33, Sevastopol, Russian Federation \\ a khao@list.ru, ba.a.kharchenko@sevsu.ru, c vladetska@rambler.ru
}

\begin{abstract}
Keywords: Operational and Technological Reliability, Internal Small-Sized Thread Processing, Technological Elements of the Module, Flexible Manufacturing Module (FMM) for Thread Processing, Simplest Flow, Markov Chain, Probability of Failure-Free Operation
\end{abstract}

\begin{abstract}
The article presents the results of research in the direction of improving technological equipment for internal threading of parts in a flexible automated production. Methods for assessing the operational and technological reliability of a flexible manufacturing module (FMM) of thread processing are considered, which can be used as the basis for the developed method of synthesis of its elements. It is proposed to consider the technological system of the flexible manufacturing module (FMM) of thread processing as a system in which transitions from state to state occur under the action of the simplest flows with parameters of the transition probabilities of a continuous Markov chain. The developed mathematical model describing the state of the FMM taking into account the failures of the functioning of its elements, parametric failures, as well as taking into account the recovery after these types of failures, makes it possible to reflect the influence on the operation of the module of the parameters of the flows of failures and restorations of the tool, machine tool, fixture, loading device. The solution of the obtained systems of equations of final probabilities allows for given (or experimentally obtained) intensities of failure streams (functioning and parametric) for FMM threading to obtain the values of the probability of failurefree operation, as well as the probabilities of finding the system in an inoperative state due to corresponding failures. The measures taken make it possible to solve the synthesis problem at the level of structural and layout optimization, so that at the stage of parametric synthesis to determine the elements that are vulnerable according to the developed criterion, the improvement of which will lead to the creation of the most efficient system.
\end{abstract}

\section{Introduction}

The creation of effective technological equipment for flexible manufacturing modules (FMM) for automated thread processing based on such modern technological elements as a machine tool, a tool, a fixture, a loading device should be carried out at the level of new requirements and is associated with obtaining an economic effect by increasing processing productivity and improving the quality of products. When creating elements of flexible manufacturing modules (FMM) for small-sized internal threading, one should take into account the effect of failure streams (parametric and functional) on the output of the system. It is necessary to describe the process of formation of indicators of operational and technological reliability as a result of their interaction in the real conditions of the operation of threading equipment. The impact of operational reliability on productivity and economic efficiency is assessed by introducing the availability factor or the technical utilization factor $[1,2]$. Obtaining this estimate is of practical interest, but it does not take into account all aspects related to parametric failures of various elements. 
In [2], a performance model is proposed based on taking into account sudden failures of elements of a technological system, assuming Poisson reliability laws, and an exponential distribution of the recovery time. At the same time, steady-state probabilities exist and the known results of the mathematical theory of queuing were used to determine them [3]. Such an approach to the construction of mathematical models is useful at the preliminary stage of system synthesis, but for assessing the efficiency of the FMM at the last stages of parametric synthesis, such an indicator is clearly insufficient due to the assumptions about the homogeneity of the system elements in terms of failures and maintenance.

Methods for analytical assessment of the technological reliability of elements are usually based on mathematical models of changes in processing accuracy over time [4, 5]. Models [1, 6] have been developed for the analysis and forecasting of reliability, which creates the possibility of finding ways to reduce the influence of errors on the change in reliability and the impact on it not only by organizational, but also by design methods. It is advisable to use this approach in the synthesis of FMM for thread processing at the stage of assessing the intensity of parametric failures of technological elements by choosing the main types of initial errors. For the processes of cutting internal threads, it is necessary to determine the main parameters of processing accuracy, to develop a methodology for their assessment.

Studies of the technological reliability of thread-cutting equipment are given in [7], where a hypothesis about the mechanism of size formation when working with a tap is proposed, according to which the profile on the product is formed as a result of the "transfer" of the tool size during cutting. The author theoretically and experimentally proved that at the achieved level of accuracy of the technological system, cutting of precise threads can be performed if it is possible to create optimal designs of taps, tap holders, bearing spindles and the machine tools themselves. The approach used by researchers [8,9] allows predicting the accuracy of threaded holes when processing various materials and developing practical recommendations. The methods for determining the precision parameters of the thread can be taken as a basis for determining the characteristics of the technological reliability of the FMM elements for thread processing.

Studies of the operational reliability of threading equipment $[10,11,12]$ have shown that as a result of breakage, $50 \ldots 70 \%$ of cutting taps with sizes M2...M6 fail. Ranking by the specific weight of failures indicates that tools are in the first place, then, respectively, mechanical units, electrical and hydraulic equipment [13]. As measures to improve the reliability of the tool, it is necessary to increase its durability, strength, pay more attention to the choice of its design, operating modes. Currently, many designs of taps and cutting patterns are known [14]. A number of studies of the process of thread rolling with chipless, plastic deforming taps (PDT) have been carried out. The difference between cutting and rolling is that in the first case, the thread is formed by cutting out the metal, and in the second - by extrusion, that is, without the formation of chips, which is one of the advantages of the rolling method, especially when processing blind threads. Research and development [15], as well as standards for chipless taps, based on the results of [11], played a significant role in the popularization and implementation of the new method. Contradictory opinions can be found on the issue of PDT reliability. Some authors do not get an increase in the durability of a rolling tool in comparison with a cutting one, while others get an increase of $10 \ldots$ 20 times. Studies [12] found that thread rolling is the more efficient, the smaller the thread size. For example, when rolling M3...M4 threads in workpieces made of viscous materials, it is possible to obtain a tenfold increase in tool life, but with an increase in diameter, the difference in resistance sharply decreases and for M10...M12 threads, no advantages in durability are observed. 
Thus, the recommendations of the researchers are mainly intended and effective for narrow areas, which makes it difficult to use them in the new conditions that are characteristic of flexible automated production. These features should be taken into account when creating PDT elements for internal threading.

\section{Main part}

FMM threading is a technical dynamic system, as it has the following features: the module consists of separate interacting subsystems; the state of the subsystems and the module as a whole changes over time; each subsystem and module as a whole is characterized by its current state, history of development $[16,17]$.

On the basis of the assumptions made, to assess the options for the selected structures, it is necessary to consider the features of the occurrence of operational failures and parametric failures, that is, the operational and technological reliability of the module. Each element of the technological system can have different recovery rates $\mu$, as well as different failure rates $\lambda$, therefore, different reliability. In this case, a more complex element with the same $\lambda$ can have a lower value of $\mu$ (lower reliability), or with the same $\mu$ a larger value of $\lambda$ than a less complex element. This must be taken into account when choosing the criteria for evaluating the effectiveness of the module.

Considering the threading module as a discrete system, let us assume that the elements fail independently of each other, and each element is restored after failure. We will assume that the initial properties of an element do not in any way affect the reliability of other elements. The moments of failure of each element form the recovery process, and by virtue of our assumptions, these processes are independent. Let us denote by $F_{k}(t)$ the distribution law of the lifetime of the $k$-th technological element. We assume that these laws have a continuous density $f_{k}(t)$ and that there is an average lifetime of a technological element $T_{k}$ and its variance $\sigma_{k}^{2}$.

Since the elements in the module system are connected in series (in terms of reliability), the failure of any element causes the system to fail. The appearance of failures in one section of time does not change the probability of occurrence of any number of failures in another section that does not intersect with the first. From these physical considerations it follows that under the above assumptions, there should be no aftereffect in the flow of failures of the technological system of the module. In addition, we assumed above that the distribution laws $F_{k}(t)$ have continuous densities. Hence it follows that $H(t)$ - the average number of failures - is continuous and the flow of failures of the module system is ordinary, that is, the probability of two failures occurring simultaneously is zero. In queuing theory, it is proved that an ordinary flow with a continuous function $H(t)$, in which there is no sequence, is the simplest non-stationary flow. This means that the probability of occurrence of $n$ failures in any section $\left(t_{1}, t_{2}\right)$ is expressed by the formula

$$
P_{n}\left(t_{1}, t_{2}\right)=\frac{\left[H\left(t_{2}\right)-H\left(t_{1}\right)\right]^{n}}{n !} e^{-\left[H\left(t_{2}\right)-H\left(t_{1}\right)\right]}
$$

and for any system of non-overlapping intervals, the events consisting in the appearance of a given number of failures at each interval are independent. So, it has been shown that under fairly general conditions the flow of failures of the technological system of the module will be the simplest non-stationary flow. It can be seen from the previous formula that the number of failures at each interval is distributed according to Poisson's law (Poisson flow with a variable parameter). This variable is the failure rate $\lambda(t)$. Then the formula Eq. 1 can be written as: 


$$
P_{n}\left(t_{1}, t_{2}\right)=\frac{\left[\int_{t_{1}}^{t_{2}} \lambda(t) d t\right]^{n}}{n !} e^{-\int_{t_{1}}^{t_{2}} \lambda(t) d t}
$$

If on the segment $(t, t+\tau)$ the function $\lambda(t)$ is approximately linear, then a good agreement for these probabilities gives the equality:

$$
\operatorname{Pn}\left(t_{1}, t_{2}\right) \approx \frac{\left[\lambda\left(t+\frac{\tau}{2}\right) \tau\right]^{n}}{n !} e^{\lambda\left(t+\frac{\tau}{2}\right) d t}
$$

If the function $\lambda(t)$ on this segment is approximately constant, $\lambda(t) \approx \lambda$, then

$$
P_{n}\left(t_{1}, t+\tau\right) \approx \frac{(\lambda \tau)^{n}}{n !} e^{-\lambda \tau}
$$

Since $\lambda(t)$ almost does not change in the sections between adjacent failures, the average time until the first failure of a technological element is expressed by the approximate formula:

$$
\overline{T p} \approx 1 / \lambda(t)
$$

As a rule, the operation of the FMM technological system continues for a long time, as a result of which the elements (machine, tool, fixture, loading device) can fail several times. So, the tool in the process of weekly operation fails on average $120 \ldots 140$ times. Under these conditions, the recovery processes become stationary and, therefore, the flow of system failures is also steadystate.

Since the recovery densities for each element tend to the limits $\lim _{t \rightarrow \infty} \mu_{k}(t)=1 / \overline{\mathrm{Tk}^{\prime}}$, the failure rate of the system has a limit $\lim _{t \rightarrow \infty} \lambda(t)=\lambda_{0}=\sum_{k=1}^{m} 1 / \overline{T k^{\prime}}$, where $m$ is the number of technological elements. In this case, the flow of the system becomes a Poisson flow with a constant parameter, that is, the simplest flow. For this flow, the probability of occurrence of $n$ failures in a section of time of duration $\tau$ does not depend on the position of this section and is expressed by the formula:

$$
P_{n}(\tau)=\frac{\left(\lambda_{0} \tau\right)^{n}}{n !}=e^{-\lambda_{0} \tau}
$$

In particular, the probability of failure-free operation of the module system during time $\tau$ is equal to $P_{0}(\tau)=e^{-\lambda_{0} \tau}$, therefore, the mean time of failure-free operation is $\bar{T}=1 / \lambda_{0}$. Since the number of failures occurring in a given time interval has a Poisson distribution, the time between failures has an exponential distribution, that is, the reliability of each technological element in the module system obeys an exponential law:

$$
F_{k}(t)=1-e^{-\lambda_{k} t}
$$

The recovery process for each element will form the simplest flow and the module system recovery flow as the sum of the simplest flows will also be the simplest flow. 
The above arguments and reasoning are quite fair when considering the operational reliability (failures of functioning) of the technological system of FMM thread processing. To analyze the technological reliability (parametric failures) of the system, it is necessary to take into account the effect of such factors as cutting forces (fast processes), wear of the tool, fixture, loading device (medium speed processes), wear of the table guides, spindle bearings, fixing elements of the fixture (slow processes).

Taking into account the above arguments, we will consider the technological system of FMM threading as a physical discrete system that can be in the states $S_{0}, S_{1}, S_{2}, S_{3}, S_{4}$. State $S_{0}$ characterizes the normal functioning of the module in the absence of failures.

In the $\mathrm{S}_{1}$ state, the replacement of a failed tool is required due to the flow of failures of its functioning with the intensity $\lambda_{1}$. In this case $\lambda_{l}=1 / \overline{T и}^{\prime}$, where $\overline{T и}^{\prime}$ is the average time between two failures of functioning, $\mathrm{min}$. The $S_{2}$ state is characterized by the failure of the machine (including the die head) due to the flow of failures with the intensity $\lambda_{2}$. In this case, $\lambda_{2}=1 \overline{T c}^{\prime}$, where $\overline{T c}^{\prime}$ is the average time between two failures in the operation of the machine, min.

In the $S_{3}$ state, it is necessary to repair the broken device due to the flow of failures with the intensity $\lambda_{3}$. In this case $\lambda_{3}=1 / \overline{T \Pi}^{\prime}$, where $\overline{T \Pi}^{\prime}$ is the average time between two failures of the device functioning, $\min$.

State $S_{4}$ is characterized by the failure of the loading device due to the flow of failures of functioning with intensity $\lambda_{4}$. In this case $\lambda_{4}=1 \overline{T_{3}}$, where $\overline{T_{3}}$ ' is the average time between two failures in the functioning of the loading device, $\min$.

The quantities $\overline{T и}^{\prime}, \overline{T c}^{\prime}, \overline{T \Pi}^{\prime}, \overline{T 3}^{\prime}$ are determined by the general formula:

$$
\overline{T^{\prime}}=\sum_{j=1}^{m} t_{j} / m
$$

where $m$ is the number of failures in the functioning of the corresponding technological element of the FMM threading; $t_{j}$ - time interval between $(j-1)$-th and $j$-th orders.

Each technological element of the module undergoes recovery immediately after failure. The recovery rate, respectively, for each technological element will be $\mu_{1}, \mu_{2}, \mu_{3}, \mu_{4}$, assuming that the recovery time, being a random variable, obeys Poisson's law.

In this case $\mu_{1}=1 / \overline{T \mathrm{Bи}}^{\prime}, \mu_{2}=1 / \overline{T \mathrm{~B} C}^{\prime}, \mu_{3}=1 / \overline{T \mathrm{B \Pi}}^{\prime}, \mu_{4}=1 / \overline{T \mathrm{~B} 3}^{\prime}$, where $\overline{T \mathrm{Bи}}^{\prime}$ - average recovery (replacement) time of the tool, $\min ; \overline{\mathrm{TBC}}^{\prime}$ - average time of restoration (repair) of machine elements, including the threading head, $\min ; \overline{T \mathrm{B \Pi}}^{\prime}$ - average recovery (repair) time of the device, $\min ; \overline{\mathrm{T}}^{\prime}$ - average recovery (repair) time of the boot device, $\min$.

To describe the labeled graph of states of the technological system of FMM threading, taking into account the failures of functioning (Fig. 1), we denote:

$P_{0}-$ is the probability of the correct operation of the module, $P_{0}=P\left(S_{0}\right)$;

$P_{1}, P_{2}, P_{3}, P_{4}$ - respectively, the probability of breakage (failure) of the tool, machine elements and threading head, fixture, loading device. 

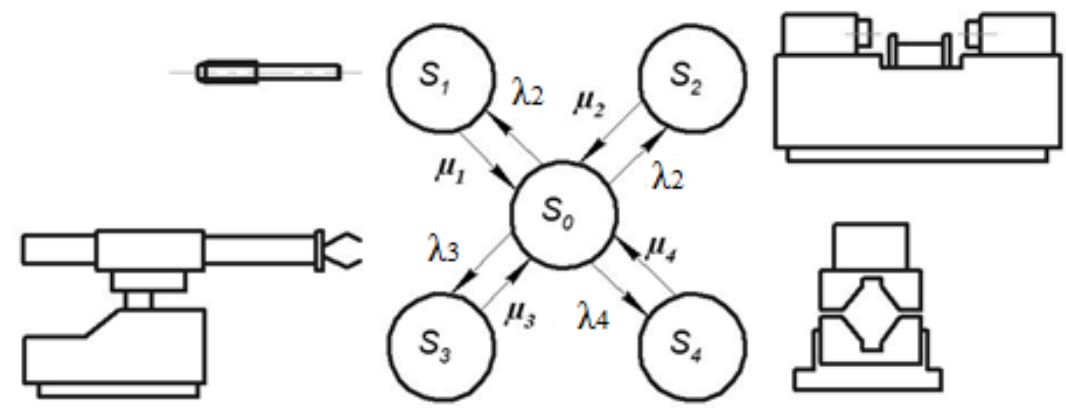

Fig. 1. The marked graph of states of the technological system of the FMM thread processing, taking into account the failures of functioning

A. Kolmogorov's equations for the final probabilities $P_{0}, P_{1}, P_{2}, P_{3}, P_{4}$ of states $S_{0}, S_{1}, S_{2}, S_{3}$, $S_{4}$ are represented as a system of equations:

$$
\left.\begin{array}{l}
P_{0}\left(\lambda_{1}+\lambda_{2}+\lambda_{3}+\lambda_{4}\right)=P_{1} \mu_{1}+P_{2} \mu_{2}+P_{3} \mu_{3}+P_{4} \mu_{4} \\
P_{1} \mu_{1}=P_{0} \lambda_{1} \\
P_{2} \mu_{2}=P_{0} \lambda_{2} \\
P_{3} \mu_{3}=P_{0} \lambda_{3} \\
P_{4} \mu_{4}=P_{0} \lambda_{4}^{\prime}
\end{array}\right\}
$$

Solving the resulting system of equations using the normalization condition $P_{0}+P_{1}+P_{2}+P_{3}+P_{4}=1$ allows one to obtain expressions for the probability of failure-free operation $\left(P_{0}\right)$, as well as the probabilities of the system being in an inoperative state due to corresponding breakdowns (failure) of the tool $\left(P_{1}\right)$, machine elements $\left(P_{2}\right)$, fixture $\left(P_{3}\right)$, loading device $\left(P_{4}\right)$ :

$$
\left.\begin{array}{l}
P_{0}=\left(1+\lambda_{1} / \mu_{1}+\lambda_{2} / \mu_{2}+\lambda_{3} / \mu_{3}+\lambda_{4} / \mu_{4}\right)^{-1} \\
P_{1}=P_{0} \lambda_{1} / \mu_{1} \\
P_{2}=P_{0} \lambda_{2} / \mu_{2} \\
P_{3}=P_{0} \lambda_{3} / \mu_{3} \\
P_{4}=P_{0} \lambda_{4}^{\prime} / \mu_{4}
\end{array}\right\}
$$

The technological system of FMM threading as a discrete system, due to parametric failures resulting from tool deformations, positioning errors of the loading device, fixture, spindle runout, changes in the temperature of the machine tool and control device elements, loses serviceability, not ensuring machining accuracy.

FMM as a result, it can be in the states $S_{00^{\prime}}, S_{10^{\prime}}, S_{20}^{\prime}, S_{30}^{\prime}, S_{01}^{\prime}, S_{02}{ }^{\prime}, S_{03^{\prime}}{ }^{\prime}, S_{11}^{\prime}, S_{21}^{\prime}, S_{31}{ }^{\prime}, S_{12}{ }^{\prime}$, $S_{22}{ }^{\prime}, S_{32}{ }^{\prime}, S_{13}{ }^{\prime}, S_{23}{ }^{\prime}, S_{33}{ }^{\prime}$.

State $S_{00}{ }^{\prime}$ characterizes the correct operation of the module in the absence of parametric failures.

In state $S_{10}{ }^{\prime}$, parametric tool failures with intensity $\lambda_{01}$ are observed. In this case $\lambda_{01}=1 / \overline{T и}^{\prime \prime}$, where $\overline{\text { й }^{\prime \prime}}$ is the average time between two parametric tool failures, $\min$.

In state $S_{20}{ }^{\prime}$, inspection and readjustment of the machine, the die head is required due to the 
flow of failures with the intensity $\lambda_{02}$. In this case, $\lambda_{02}=1 \overline{T \mathrm{c}^{\prime \prime}}, \overline{T \mathrm{c}^{\prime \prime}}$ is the average time between two parametric machine failures, $\min$.

State $S_{30}{ }^{\prime}$ is characterized by the simultaneous presence of parametric failure streams for tool $\lambda_{01}$ and machine tool $\lambda_{02}$.

In state $S_{01}{ }^{\prime}$, inspection and readjustment of the device is required with the intensity of incoming parametric failures $\lambda_{01}^{\prime}$. In this case $\lambda_{01}^{\prime}=1 \overline{T_{\Pi^{\prime \prime}}}$, where $\overline{T_{\Pi^{\prime \prime}}}$ is the average time between two parametric failures of the device, $\min$.

The state $S_{02}$ is characterized by the presence of parametric failures of the loading device due to the flow with the intensity of requests $\lambda_{02}^{\prime}$. In this case $\lambda_{02}^{\prime}=1 \overline{T_{3}^{\prime \prime}}$, where $\overline{T 3}^{\prime \prime}$ is the average time between two parametric failures of the loading device, min.

In the $S_{03}{ }^{\prime}$ state, inspection and readjustment of the device and the loading device is required due to the flows of incoming parametric failures with intensities $\lambda_{01}^{\prime}$ and $\lambda_{02}^{\prime}$.

State $S_{11}{ }^{\prime}$ is characterized by the simultaneous presence of streams of parametric failures of the tool with the intensity $\lambda_{01}$ and the fixture with the intensity $\lambda_{01}^{\prime}$.

In the state $S_{21}{ }^{\prime}$, inspection and readjustment of the machine and the fixture is required, caused, respectively, by the flows of failures with the rates $\lambda_{02}^{\prime}$ and $\lambda_{01}^{\prime}$.

State $S_{31}{ }^{\prime}$ is characterized by the simultaneous presence of streams of parametric failures of tool $\lambda_{01}$, machine tool $\lambda_{02}$ and fixture $\lambda_{01}^{\prime}$.

In the $S_{12}$ state, inspection and readjustment of the tool and the loading device is required due to the flows of parametric failures with intensities $\lambda_{01}$ and $\lambda_{02}^{\prime}$.

State $S_{22}{ }^{\prime}$ is characterized by the simultaneous presence of parametric machine failures with intensity $\lambda_{02}$ and a loading device with intensity $\lambda_{02}^{\prime}$.

In the $S_{32}$ state, inspection and readjustment of the tool, machine tool and loading device is required, caused, respectively, by the failure streams with rates $\lambda_{01}, \lambda_{02}$ and $\lambda_{02}^{\prime}$.

State $S_{13}{ }^{\prime}$ is characterized by the presence of streams of parametric failures of the tool with the intensity $\lambda_{01}$, the fixture with the intensity $\lambda_{01}^{\prime}$ and the loading device with the intensity $\lambda_{02}^{\prime}$.

In the $S_{23}$ ' state, inspection and readjustment of the machine, the fixture and the loading device is required due to flows of parametric failures with intensities $\lambda_{02}, \lambda_{01}^{\prime}$ and $\lambda_{02}^{\prime}$, respectively.

State $S_{33}{ }^{\prime}$ is characterized by the presence of streams of parametric failures of a tool with an intensity $\lambda_{01}$, a machine tool with an intensity $\lambda_{02}$, a fixture with an intensity $\lambda_{01}^{\prime}$ and a loading device with an intensity $\lambda_{02}^{\prime}$.

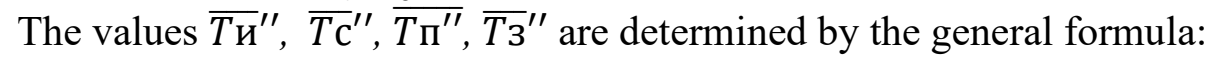

$$
\overline{T^{\prime \prime}}=\sum_{j=1}^{k} t_{j} / k
$$

where $k$ is the number of parametric failures of the corresponding technological element during the operation of the FMM for thread processing; $t_{j}$ - time interval between $(j-1)$-th and $j$-th failures.

Each technological element of the FMM thread processing undergoes restoration immediately after parametric failure. Recovery rates, respectively, for each technological element $\mu_{01}, \mu_{02}, \mu_{01}^{\prime}$,

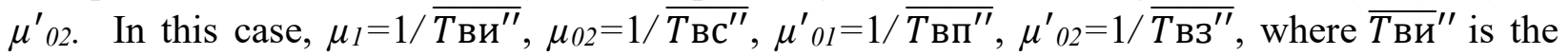
average recovery time (inspection and adjustment) of the tool, $\min ; \overline{\mathrm{TBC}}^{\prime \prime}$ - average time of inspection and adjustment of the machine, $\min ; \overline{\mathrm{TB}^{\prime \prime}}$ - average time of inspection and adjustment of the device, $\min ; \overline{T \text { вз }}^{\prime \prime}$ - average time of inspection and adjustment of the loading device, $\min$.

To describe the marked-up graph of states of the FMM technological system of thread 
processing, taking into account parametric failures (Fig. 2), the following designations are adopted:

$P_{00}{ }^{\prime}$ - the probability of the module operation in the absence of parametric failures, $P_{00}{ }^{\prime}=$ $P\left(S_{00}{ }^{\prime}\right)$;

$P_{10}{ }^{\prime}, P_{20}{ }^{\prime}, P_{30}{ }^{\prime}-$ respectively, the probability of parametric failures of the tool, machine, tool and machine at the same time;

$P_{01}{ }^{\prime}, P_{02}{ }^{\prime}, P_{03}{ }^{\prime}$ - the probabilities of parametric failures, respectively, of the device, the loading device, the device and the loading device at the same time;

$P_{11}^{\prime}, P_{21}^{\prime}, P_{31}^{\prime}-$ respectively, the probability of parametric failures of the tool and fixture; machine tool and fixtures; tool, machine and fixture at the same time;

$P_{12}{ }^{\prime}, P_{22}{ }^{\prime}, P_{32}{ }^{\prime}$ - the probabilities of parametric failures of the tool and the loading device; machine and loading device; tool, machine and loading device;

$P_{13}{ }^{\prime}, P_{23}{ }^{\prime}, P_{33}{ }^{\prime}-$ the probabilities of parametric failures of the tool of the fixture and the loading device; machine tool, fixture and loading device; simultaneously all technological elements - tool, machine tool, fixture and loading device.

Equations for final probabilities $P_{00}{ }^{\prime}, P_{10}{ }^{\prime}, P_{20}{ }^{\prime}, P_{30}{ }^{\prime}, P_{01}{ }^{\prime}, P_{02}{ }^{\prime}, P_{03^{\prime}}{ }^{\prime}, P_{11}{ }^{\prime}, P_{21}{ }^{\prime}, P_{31}{ }^{\prime}, P_{12}{ }^{\prime}, P_{22}{ }^{\prime}$, $P_{32}{ }^{\prime}, P_{13}{ }^{\prime}, P_{23}{ }^{\prime}, P_{33}{ }^{\prime}$ states are represented as a system of equations:

$$
\begin{aligned}
& P_{00}\left(\lambda_{01}+\lambda_{02}+\lambda^{I}{ }_{01}+\lambda^{I}{ }_{02}\right)=P_{10}{ }^{I} \mu_{01}+P_{20}{ }^{I} \mu_{02}+P_{01}{ }^{I} \mu^{I}{ }_{01}+P_{02}{ }^{I} \mu^{I}{ }_{02} \\
& P_{10}{ }^{I}\left(\lambda_{02}+\lambda^{I}{ }_{01}+\lambda^{I}{ }_{02}+\mu_{01}\right)=P_{00}{ }^{I} \lambda_{01}+P_{30}{ }^{I} \mu_{02}+P_{11}{ }^{I} \mu^{I}{ }_{01}+P_{12}{ }^{I} \mu^{I}{ }_{02} \\
& P_{20}{ }^{I}\left(\lambda_{01}+\lambda^{I}{ }_{01}+\lambda^{I}{ }_{02}+\mu_{02}\right)=P_{00}{ }^{I} \lambda_{02}+P_{30}{ }^{I} \mu_{01}+P_{21}{ }^{I} \mu^{I}{ }_{01}+P_{22}{ }^{I} \mu^{I}{ }_{02} \\
& P_{30}{ }^{I}\left(\lambda^{I}{ }_{01}+\lambda^{I}{ }_{02}+\mu_{01}+\mu_{02}\right)=P_{20}{ }^{I} \lambda_{01}+P_{10}{ }^{I} \lambda_{02}+P_{31}{ }^{I} \mu^{I}{ }_{01}+P_{32}{ }^{I} \mu_{02} \\
& P_{01}{ }^{I}\left(\lambda_{01}+\lambda_{02}+\lambda^{I} 02+\mu^{I}{ }_{01}\right)=P_{11}{ }^{I} \mu_{01}+P_{21}{ }^{I} \mu_{02}+P_{00}{ }^{I} \lambda^{I}{ }_{01}+P_{03}{ }^{I} \mu^{I} 02 \\
& P_{11}{ }^{I}\left(\lambda_{02}+\lambda^{I}{ }_{02}+\mu^{I}{ }_{01}+\mu_{01}\right)=P_{01}{ }^{I} \lambda_{01}+P_{10}{ }^{I} \lambda^{I}{ }_{01}+P_{31}{ }^{I} \mu_{02}+P_{13}{ }^{I} \mu^{I}{ }_{02} \\
& P_{21}{ }^{I}\left(\lambda_{01}+\lambda^{I} 02+\mu_{02}+\mu^{I}{ }_{01}\right)=P_{01}{ }^{I} \lambda_{02}+P_{20}{ }^{I} \lambda^{I}{ }_{01}+P_{31}{ }^{I} \mu_{01}+P_{23}{ }^{I} \mu^{I} 02 \\
& P_{31}{ }^{I}\left(\lambda^{I}{ }_{02}+\mu_{01}+\mu_{02}+\mu^{I}{ }_{01}\right)=P_{30}{ }^{I} \lambda^{I} 01+P_{21}{ }^{I} \lambda_{01}+P_{11}{ }^{I} \lambda_{02}+P_{33}{ }^{I} \mu^{I}{ }_{02} \\
& P_{02}{ }^{I}\left(\lambda_{01}+\lambda^{I}{ }_{01}+\lambda_{02}+\mu^{I}{ }_{02}\right)=P_{00}{ }^{I} \lambda^{I}{ }_{02}+P_{03}{ }^{I} \mu^{I}{ }_{01}+P_{22}{ }^{I} \mu_{02}+P_{12}{ }^{I} \mu_{01} \\
& P_{12}{ }^{I}\left(\lambda^{I} 01+\lambda_{02}+\mu_{01}+\mu^{I}{ }_{02}\right)=P_{02}{ }^{I} \lambda_{01}+P_{10}{ }^{I} \lambda^{I}{ }_{02}+P_{32}{ }^{I} \mu_{02}+P_{13}{ }^{I} \mu^{I}{ }_{01} \\
& P_{22}{ }^{I}\left(\lambda_{01}+\lambda^{I} 01+\mu_{02}+\mu^{I}{ }_{02}\right)=P_{02}{ }^{I} \lambda_{02}+P_{20}{ }^{I} \lambda^{I} 02+P_{32}{ }^{I} \mu_{01}+P_{23}{ }^{I} \mu^{I}{ }_{01} \\
& P_{32}{ }^{I}\left(\lambda^{I}{ }_{01}+\mu_{01}+\mu_{02}+\mu^{I}{ }_{02}\right)=P_{22}{ }^{I} \lambda_{01}+P_{12}{ }^{I} \lambda_{02}+P_{30}{ }^{I} \lambda^{I} 02+P_{33}{ }^{I} \mu^{I}{ }_{01} \\
& P_{03}{ }^{I}\left(\lambda_{01}+\lambda_{02}+\mu^{I}{ }_{01}+\mu^{I}{ }_{02}\right)=P_{02}{ }^{I} \lambda^{I}{ }_{01}+P_{01}{ }^{I} \lambda^{I}{ }_{02}+P_{13}{ }^{I} \mu_{01}+P_{23}{ }^{I} \mu_{02} \\
& P_{13}{ }^{I}\left(\lambda_{02}+\mu_{01}+\mu^{I}{ }_{01}+\mu^{I} 02\right)=P_{03}{ }^{I} \lambda_{01}+P_{11}{ }^{I} \lambda^{I}{ }_{02}+P_{12}{ }^{I} \lambda^{I}{ }_{01}+P_{33}{ }^{I} \mu_{02} \\
& P_{23}{ }^{I}\left(\lambda_{01}+\mu_{02}+\mu^{I}{ }_{01}+\mu^{I}{ }_{02}\right)=P_{03}{ }^{I} \lambda_{02}+P_{22}{ }^{I} \lambda^{I} 01+P_{21}{ }^{I} \lambda^{I} 02+P_{33}{ }^{I} \mu_{01} \\
& P_{33}{ }^{I}\left(\mu_{01}+\mu_{02}+\mu^{I}{ }_{01}+\mu^{I}{ }_{02}\right)=P_{23}{ }^{I} \lambda_{01}+P_{13}{ }^{I} \lambda_{02}+P_{32}{ }^{I} \lambda^{I} 01+P_{31}{ }^{I} \lambda^{I}{ }_{02}
\end{aligned}
$$




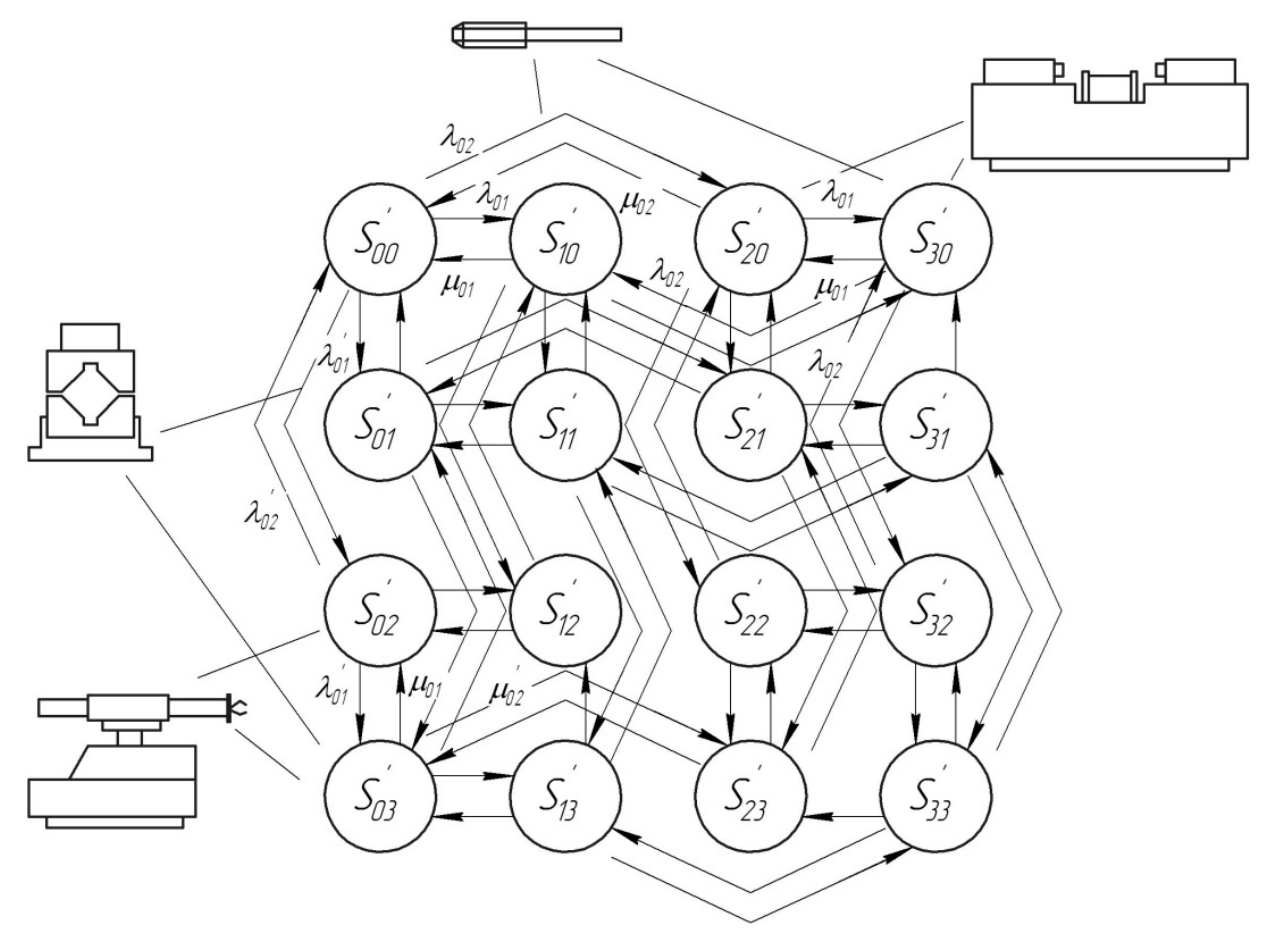

Fig. 2. The marked graph of states of the FMM technological system of thread processing, taking into account parametric failures

Solving this system of equations using the normalization condition $P_{00}{ }^{\prime}+P_{10}{ }^{\prime}+P_{20}{ }^{\prime}+P_{30}{ }^{\prime}+$ $P_{01}{ }^{\prime}+P_{02}{ }^{\prime}+P_{03}{ }^{\prime}+P_{11}{ }^{\prime}+P_{21}{ }^{\prime}+P_{31}{ }^{\prime}+P_{12}{ }^{\prime}+P_{22}{ }^{\prime}+P_{32}{ }^{\prime}+P_{13}{ }^{\prime}+P_{23}{ }^{\prime}+P_{33}{ }^{\prime}=1$ allows you to obtain expressions for the probability of failure-free parametric operation $\left(P_{00^{\prime}}\right)$, as well as the probabilities of finding the system in a faulty state due to the corresponding parametric failures of one or more (in different combinations) technological elements of the FMM threading (tool $-P_{10}{ }^{\prime}$, machine elements $-P_{20}{ }^{\prime}$, fixtures $-P_{01}{ }^{\prime}$, loading device $-P_{02}{ }^{\prime}$ ).

\section{Summary}

The carried out theoretical studies made it possible to develop a mathematical model for describing the states of the FMM thread processing, taking into account the failures of functioning and parametric failures of its technological elements, as well as recoveries after these failures. The model makes it possible to take into account the operational and technological reliability of the tool, the power threading head, the basic elements of the machine, the fixture, the loading device when choosing the optimal structure of the module.

The structure of the model and the labeled graph of the states of the system can be improved as the number of parameters and characteristics is refined. For complete information and an objective assessment of the preferred option for use in FMM conditions, it is necessary to take into account the stochastic processes occurring in the system under real operating conditions, as well as to develop an optimality criterion and an integral evaluation function, taking into account, in addition to reliability (operational and technological), flexibility (adaptability) and economic efficiency of the module [18-22].

The measures taken make it possible to solve the synthesis problem at the level of structural and layout optimization, and then, at the stage of parametric synthesis, to determine technological 
elements that are vulnerable according to the developed criterion, the improvement of which will lead to the creation of the most efficient system.

\section{References}

[1] A.S. Pronikov, Metal-cutting machines and automatic machines, Mashinostroyeniye, Moscow, 1981, 479 p.

[2] V.E. Push, R. Pigert, V.L. Sosonkin, Automatic machine tools, Mashinostroyeniye, Moscow, 1982, 319 p.

[3] E.S. Wentzel, L.A. Ovcharov, Probability Theory and Its Engineering Applications, Vysshaya shkola, Moscow, 2000, 480 p.

[4] G. D. Grigoryan, Elements of reliability of technological processes, Vishcha school, Vishcha shkola, Kiev-Odessa, 1984, 214 p.

[5] A.A. Matalin, Precision Machining and Process Design, Mashinostroyeniye, Moscow, 1970, $380 \mathrm{p}$.

[6] A.S. Pronikov, Precision and reliability of numerical control machines, Mashinostroyeniye, Moscow, 1982, 279 p.

[7] V.V. Matveyev, Precision thread cutting, Mashinostroyeniye, Moscow, 1978, 88 p.

[8] Yu.L. Frumin, High Performance Threading Tool, Mashinostroyeniye, Moscow, 1977, 183 p.

[9] A.S. Yamnikov, Determination of dynamic errors in threading with a measuring tool, Research in the field of technology for the formation of external and internal threads, threadforming tools, machine tools and methods for controlling threads, Tula: Publisher TPI, 1974. pp. 68-72.

[10] A.A. Grudov, Ways to increase the accuracy of the cut thread and the durability of taps, Publisher VNII, Moscow, 1971. - 128 p.

[11] V.M. Menshakov, G.P. Urlapov, V.S. Sereda, Chipless taps, Mashinostroyeniye, Moscow, 1976. $-167 \mathrm{p}$.

[12] V.G. Yakukhin, Optimal thread manufacturing technology, Mashinostroyeniye, Moscow, 1976, 167 p.

[13] G.I. Melamed, B.M. Tursunov, Flexible automatic production. CNC machines and robots, Publisher Belarus, Minsk, 1986, 159 c.

[14] S.M. Bratan, F.N. Kanareyev, P.A. Novikov, A.O. Kharchenko, Improving the accuracy of forming small-sized threads with taps in aluminum alloys, Vuzovskiy uchebnik: INFRA-M, Moscow, 2017, 164 p.

[15] E.V. Ryzhov, O.S. Andreychikov, A.E. Steshkov, Thread rolling, Mashinostroyeniye, Moscow, 1974, 122 p.

[16] Yu.K. Novoselov, A.O. Kharchenko, Choice of the Optimal Structure of a Flexible Production Cell, Soviet engineering research. 7(2) (1987), pp. 48-52.

[17] A.O. Kharchenko, Development of flexible production modules taking into account the reliability of technological systems, Publisher Society " Znaniye", Kiev, 1989, 20 p. 
[18] E.G. Nahapetyan, Diagnostics of flexible automated manufacturing equipment, Publisher Nauka, Moscow, 1985, 224 p.

[19] A.O. Kharchenko, S.M. Bratan, E.A. Vladetskaya, S.I. Roshchupkin, Analysis and Synthesis of Structures of Modern Multi-Operational Machine Tools, Publisher Tsentrkatalog, Moscow, 2018, 144 p.

[20] V.P. Dolgin, A.O. Kharchenko, Reliability of technical systems, Publisher Vuzovskiy uchebnik, INFRA-M, Moscow, 2015, 157 p.

[21] A.O. Kharchenko, A.A. Kharchenko, Structural and layout synthesis of subsystems of modules for internal thread processing, Fundamental and applied problems of engineering and technology, Orel: Publisher "OSU named after I.S. Turgenev". 4-2 (342) (2020), pp. 85-94. https://doi.org/10.33979/2073-7408-2020-342-4-2-85-94

[22] A. Kharchenko, Progressive threading of small holes based on mechanisms of parallel structures (MPS), Materials Today: Proceedings, Volume 38, Part 4. (2021), pp. 2038-2042. https://doi.org/10.1016/j.matpr.2020.10.040 